\title{
The Breakage-Fusion-Bridge Cycle Producing $M L L$ Amplification in a Case of Myelodysplastic Syndrome
}

\author{
Lan Ta, ${ }^{1}$ Adrian Zordan, ${ }^{1}$ Bruce Mercer, ${ }^{1}$ Lynda J. Campbell, ${ }^{1,2}$ and Ruth N. MacKinnon ${ }^{1,2}$ \\ ${ }^{1}$ Victorian Cancer Cytogenetics Service, St Vincent's Hospital, P.O. Box 2900, Fitzroy, VIC 3065, Australia \\ ${ }^{2}$ Department of Medicine, St Vincent's Hospital, University of Melbourne, Fitzroy, VIC 3065, Australia \\ Correspondence should be addressed to Ruth N. MacKinnon; ruth.mackinnon@svhm.org.au
}

Received 23 May 2013; Accepted 24 June 2013

Academic Editor: Mario Scartozzi

Copyright (c) 2013 Lan Ta et al. This is an open access article distributed under the Creative Commons Attribution License, which permits unrestricted use, distribution, and reproduction in any medium, provided the original work is properly cited.

\begin{abstract}
Telomere loss may lead to chromosomal instability via the breakage-fusion-bridge (BFB) cycle which can result in genetic amplification and the formation of ring and dicentric chromosomes. This cycle continues until stable chromosomes are formed. The case of a 72-year-old female with refractory anaemia with excess blasts type 2 illustrates these events. Conventional cytogenetics produced a complex karyotype which included unstable abnormalities of chromosomes 11, 12, and 15. Fluorescence in situ hybridization (FISH) analyses including multicolor-FISH (M-FISH) and multicolor-banding (M-BAND) revealed multiple clonal populations with 5 copies of $M L L$ on either a ring chromosome composed entirely of chromosome 11 material or a derivative chromosome composed of chromosomes 11, 12, and 15. The FISH results also clarified the likely evolution of the karyotypic complexity. The simplest cell line contained a dic $(12 ; 15)$ in addition to copy number aberrations that are typical of MDS or AML. As the disease progressed, a ring 11 was formed. Subsequently, the ring 11 appears to have unwound and inserted itself into the $\operatorname{dic}(12 ; 15)$ chromosome followed by an inversion of the derivative chromosome, producing a der(11;15;12). Telomeric loss and BFB cycles appear to have played an important role in the chromosomal rearrangements and clonal evolution demonstrated in the karyotype.
\end{abstract}

\section{Introduction}

Telomeres are composed of repetitive G-rich sequences and proteins that form a dynamic cap to maintain chromosome stability as well as prevent the chromosome ends from degradation and fusion [1]. Telomere loss may lead to the formation of ring and dicentric chromosomes, causing chromosomal instability and genetic amplification via the breakage-fusionbridge (BFB) cycle $[1,2]$. The BFB cycle can be initiated when a chromosome has lost a telomere and is replicated. The ends of the sister chromatids without a telomere may fuse together. A bridge is then formed which breaks during anaphase as the sister chromatids are pulled to opposite poles (Figure 1) [3]. This breakage often occurs very close to the site of fusion $[1,4-6]$. The chromatid with the duplication may continue undergoing $\mathrm{BFB}$ cycles until a new telomere is acquired to improve stability $[4,7]$. A nonhomologous or microhomology mediated end-joining mechanism has been implicated in the repair of broken chromosome ends that is integral to this process $[4,6,8,9]$.

MLL amplification is found in less than $1 \%$ of acute myeloid leukaemia (AML) and myelodysplastic syndromes (MDS) with an abnormal karyotype [10,11]. After MYC, it is the second most commonly amplified gene seen in these disorders $[10,11]$ and has a high correlation with TP53 inactivation or mutation [10]. MLL amplification is associated with an aggressive clinical course and extremely poor prognosis with the average rate of survival between 2 and 3 months [10].

Dicentric chromosomes are seen in many malignant disorders [12]. Dicentric chromosomes with a distance greater than $34 \mathrm{Mb}$ between the two centromeres appear to be highly unstable, whereas those with an intercentromeric distance less than $15 \mathrm{Mb}$ are more stable $[13,14]$. Unstable chromosomes are likely to undergo secondary events such as inversions, to bring the centromeres closer together, 


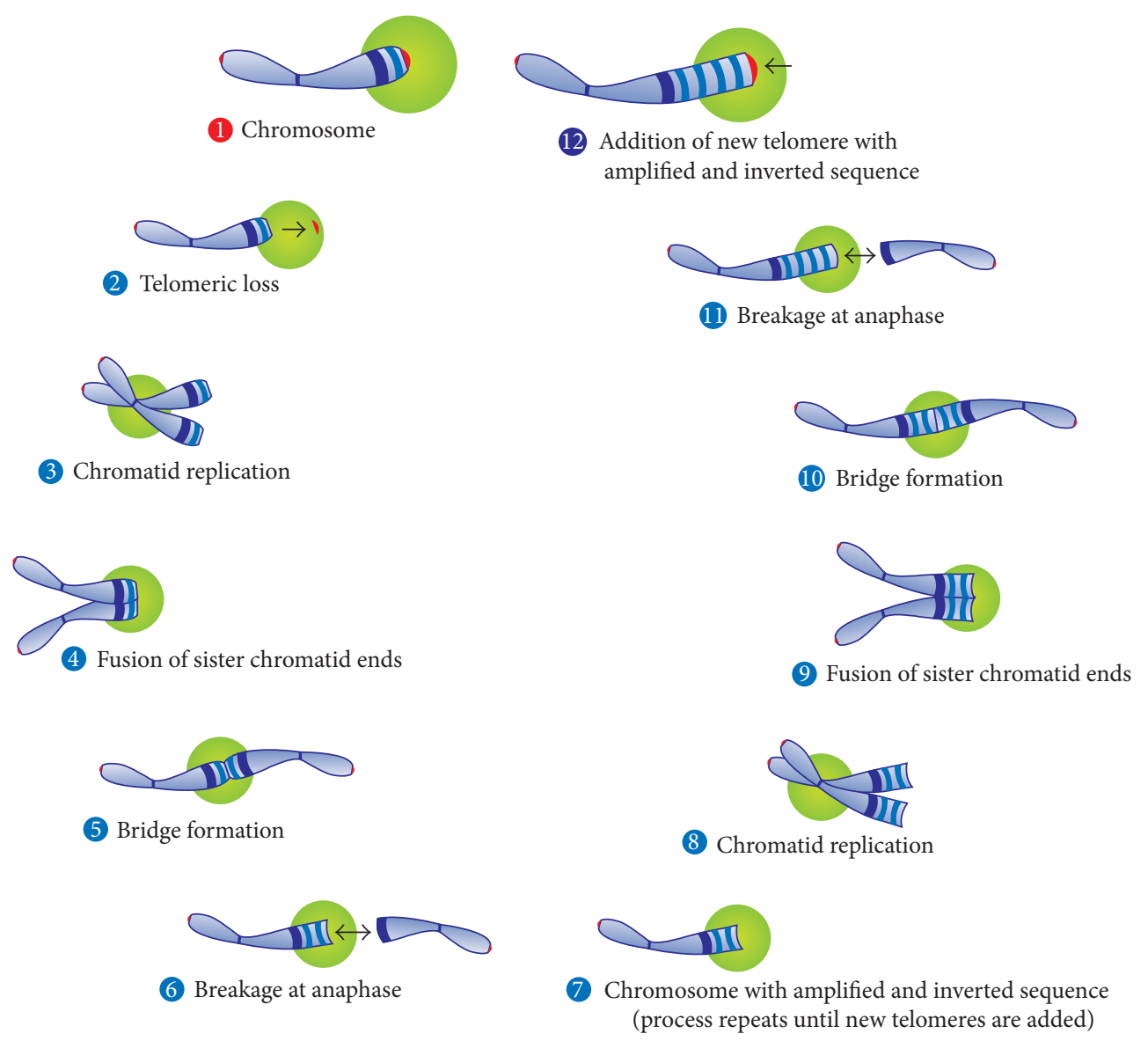

FIGURE 1: Telomeric loss leading to genetic amplification through breakage-fusion-bridge cycles. Adapted from Bailey and Murnane [1].

deletions resulting in loss of a centromere, or inactivation of a centromere, as a means of stabilisation [13-16].

We present a case of MDS transforming to AML that illustrates the role of BFB cycles in ring formation, dicentric chromosome instability, and MLL amplification.

\section{Case Presentation}

A 72-year-old woman presented with a febrile illness due to a severe chest infection. On examination, she was found to have hepatosplenomegaly and petechiae, and full blood examination showed a profound pancytopenia with haemoglobin $82 \mathrm{~g} / \mathrm{L}$, neutrophil count $0.5 \times 10^{9} / \mathrm{L}$, monocyte count $0.9 \times$ $10^{9} / \mathrm{L}$, and platelet count $30 \times 10^{9} / \mathrm{L}$. A bone marrow biopsy was performed, and a diagnosis of refractory anaemia with excess blasts type 2 (RAEB-2) (16\% blasts) was made.

Following 6 weeks of supportive care, a repeat bone marrow examination was performed, and at this time, she was diagnosed with AML with $41 \%$ blast cells identified. She received palliative care and died 6 weeks later, three months after her initial presentation.

\section{Materials and Methods}

3.1. Cytogenetics. The bone marrow specimens at diagnosis and at transformation were cultured and chromosomes were harvested and G-banded using standard techniques. Conventional cytogenetic analysis was performed, and results were described in accordance with the International System for Human Cytogenetics Nomenclature (2009) [17].

3.2. Fluorescence In Situ Hybridisation (FISH) Analysis. FISH was carried out according to the manufacturers' instructions on both the diagnostic and follow-up bone marrow samples using the Vysis LSI MLL Dual Color, Break Apart Rearrangement Probe (Abbott Molecular, IL, USA) and Vysis CEP 12 (D12Z3) SpectrumOrange Probe for the 12 centromere (Abbott Molecular). M-BAND was carried out on both samples using probes for chromosomes 11, 12 and 15 (XCyte 11, XCyte 12, and XCyte 15, Metasystems, Altlussheim, Germany). M-FISH (XCyte 24, Metasystems) was completed on the diagnostic sample only. A Zeiss Axioplan 2 microscope and an Isis image analysis system (Metasystems) were used to capture and analyse metaphase spreads.

\section{Results and Discussion}

4.1. Cytogenetics. The results of cytogenetic analysis of the diagnostic specimen are shown in Table 1.

The sample analysed after transformation to AML had the same clonal populations as the diagnostic sample. 
TABLE 1: The karyotype before and after FISH. Elements of the karyotype that are different after FISH are in bold type.

\begin{tabular}{|c|c|}
\hline Karyotype at diagnosis (RAEB-2) by G-band analysis & Revised karyotype at diagnosis after FISH, M-FISH and M-BAND analysis \\
\hline $\begin{array}{l}\text { 43 44,XX,add(5)(q13),-7,dic(12;15)(p12;p11.2), } \\
-18,+\mathbf{r 1}[\mathrm{cp} 6] / 43 \sim 44, \mathrm{sl}, \mathbf{d e r}(\mathbf{1 1}) \mathbf{r}(\mathbf{1 1} ; \text { ?)(p15q23;?)[cp8]/42, } \\
\text { sl,-11,-dic(12;15),+der(12;11;?)(12qter->12p13::11p15-> } \\
\text { 11q25::?::15q11.2->15qter),-r1[4]/86,sdl1x2 [3] }\end{array}$ & $\begin{array}{l}\text { 43,XX,del(5)(q14q35),-7,dic(12;15)(p13.2;p11.2),-18[2]/43,sl,r(11)[7]/43 44,sdl1, } \\
\text {-dic(12;15),+r(12),+der(15)(12qter->12q13::15q15->15q?11.2::12p12.3-> } \\
\text { 12p13::15p10->15q10::12q13->12q12::15q15->15qter)[cp4]/43 44,sdl1, } \\
\text {-dic(12;15),+der(12)t(12;15)(p13;q22.3),+r(15)[cp3]/43 44,sl, } \\
\text {-dic(12;15),+r(12),+der(15)[cp3]/43 44,sl,-dic(12;15),+der(12),+r(15)[cp3]/42,sl, } \\
\text {-11,+der(11;15;12)(15qter->15q14::11q22.2->11q23.3::11q22.1->11q25::11q25-> } \\
\text { 11p15.5::11q?23.3->11q?23.1::15p11.1->15q14::11q?24->11q?25::12p?13->12qter), } \\
\text {-dic(12;15)[4]/86,sdl1x2[3].ish r(11)(MLLx5),der(11;15;12) } \\
\text { (MLLx5,D12Z3+) }\end{array}$ \\
\hline
\end{tabular}

4.2. FISH, M-FISH, and M-BAND. Figure 2 outlines the chromosomes 11,12 , and 15 abnormalities in the numerous cell lines observed in the patient's bone marrow sample, including hypothetical cell lines which were not observed, but which we postulate were necessary steps to result in the final karyotype.

M-FISH revealed that the $\operatorname{der}(11) r(11 ; ?)$ was composed entirely of chromosome 11 material and was thus a ring 11 (Figure 2(b)). It also exposed the complexity of the rearrangements occurring between chromosomes 12 and 15 resulting in multiple clones which included either a dic(12;15), a derivative 12 chromosome with an additional ring 15 , or a derivative 15 chromosome with a ring 12 . In addition, the $\operatorname{der}(12 ; 11 ;$ ?) contained material from chromosomes 11,12 , and 15 , including the pericentric region of chromosome 15.

FISH results showed five copies of $M L L$ on each of the ring 11 and the der(12;11;?) (Figure 3 ). The 12 centromere was present on the der $(12 ; 11 ; ?)$, but inactivation of the 12 centromere was evidenced by the separation of the chromatids with no centromeric constriction.

M-BAND with the XCyte 11, 12, and 15 probes provided additional information on these abnormalities and the sequence of events which may have unfolded, as well as confirming the results of M-FISH. The dic(12;15) showed no obvious loss of chromosomes 12 or 15 material, consistent with a telomere fusion event between $12 p$ and $15 p$. MBAND also demonstrated that the $\operatorname{dic}(12 ; 15)$ in the initial cell line had rearranged, forming multiple clonal populations. In some cells, material from chromosome 15, including the centromere, had spliced out of the $\operatorname{dic}(12 ; 15)$ to form a ring 15 leaving behind a derivative 12 which apparently contained all of chromosome 12 and the remainder of 15q (Figure 2(c)). In other cells, material from chromosome 12, including the 12 centromere, spliced out of the $\operatorname{dic}(12 ; 15)$ to form a ring 12 , and there was inversion of the remaining chromosomes 12 and 15 material in the derivative 15 (Figure 2(d)). These variations in the derivative chromosomes were seen in clones which contained normal chromosomes 11 as well as the ring 11 (Figures 2(e) and 2(f)), suggesting that excision of the ring 12 and ring 15 occurred on multiple occasions.

M-BAND also showed that the $\operatorname{der}(12 ; 11 ;$ ?) was a der(11;15;12), with an inversion occurring between chromosomes 11 and 15 and five copies of band 11q23 present, confirming five copies of $M L L$ as shown by FISH (Figures 2(g) and 3).
M-BAND analysis provided information on the possible mechanism of formation of the der(11;15;12). It seems likely that the ring 11 underwent breakage, causing it to unwind and insert into the dic $(12 ; 15)$ chromosome in the 15 q14 region (Figure 2(g)). Breakage of these two unstable chromosomes in a single cell would have allowed the linearized ring 11 to insert into the $\operatorname{dic}(12 ; 15)$, providing it with two telomeres. The increased instability of the resultant chromosome led to the observed inversion between chromosomes 11 and 15, presumably reducing instability [13], following which the still unstable chromosome was further stabilised by inactivation of 12 and 15 centromeres to produce a stable, functionally monocentric chromosome. This is evidenced by loss of centromeric constrictions in the 12 and 15 centromeric regions and a positive signal using the 12 centromere probe.

Telomeres gradually shorten over time, a process which can contribute to ageing, but are replenished in order for cell division to occur [18]. Telomeric loss and telomeric association resulting in BFB cycles play an important role in the formation and rearrangements of ring and dicentric chromosomes as well as the development of genetic amplification $[1,19]$. Therefore, telomeric loss may have been an important contributing factor to the various cell populations observed and thus the outcome of this patient.

Gisselsson et al. [19] found that chromosomes which were involved in anaphase bridge configurations were also involved in a number of other structural rearrangements and that BFB cycles were the mechanism for producing heterogeneity by chromosomal remodelling, such as genetic amplification. Most of the evidence that telomere erosion produces BFB cycles has come from animal models and in vitro studies. However, recent studies have produced evidence that telomere shortening causes chromosome fusion and ring formation [6, 20], chromosome instability [21], and BFB cycles [22] in human malignancy, particularly haematological malignancy [3].

BFB events are the likely mechanisms for the generation of multiple cell lines in this case study. The advanced age of the patient or prior exposure to toxins, known to be associated with telomere degradation [23], may be the mechanism for the formation of both the $\operatorname{dic}(12 ; 15)$ and ring 11 in our case, via the production of sticky ends. Although telomeric association was not observed, it is possible that this was a contributing factor. 


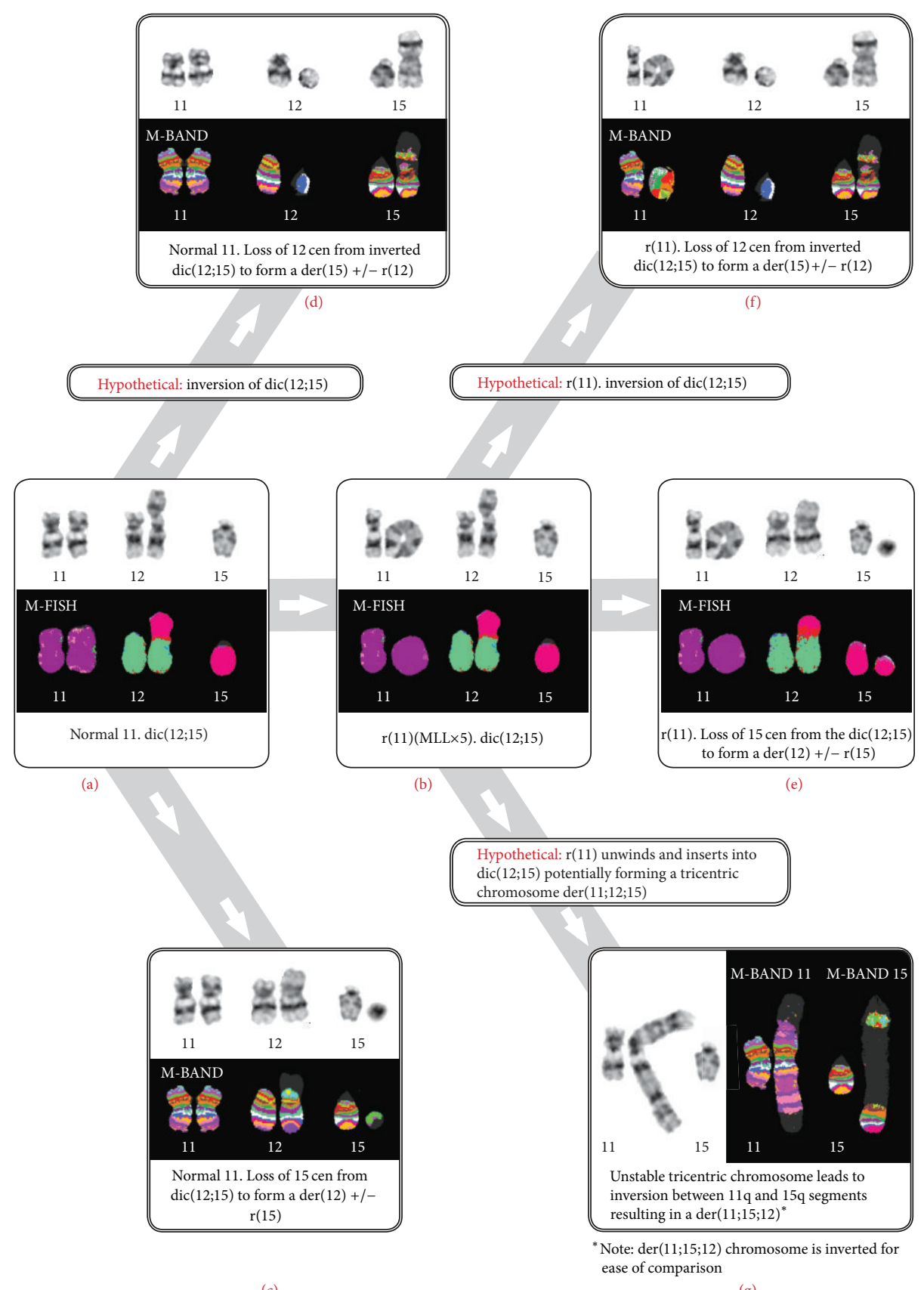

(c)

(g)

FIGURE 2: Proposed evolution of cell lines showing (a) stem line with two normal chromosomes 11 and a dic(12;15), (b) formation of $r(11)$ with $\operatorname{dic}(12 ; 15)$ present, (c) two normal chromosomes 11 with chromosome 15 material excised from the dic(12;15) to form a der(12) +/- r(15), (d) two normal chromosomes 11 with an inversion occurring in the dic(12;15) and excision of chromosome 12 material to form a der(15) +/$r(12)$, (e) $r(11)$ with chromosome 15 material excised from the dic (12;15) to form a der(12) $+/-r(15)$, (f) $r(11)$ with an inversion occurring in the $\operatorname{dic}(12 ; 15)$ and excision of chromosome 12 material to form a der(15) $+/-\mathrm{r}(12)$, and $(\mathrm{g})$ the formation of the tricentric chromosome der(11;15;12). +/- represents that the cell population is a composite and the ring was observed on at least 2 occasions but was not present in all cells of the population.

The copy number aberrations in the most primitive clone (deletion of $5 \mathrm{q}$ and monosomies of 7 and 18) were typical of MDS/AML. The additional abnormalities, involving chromosomes 11, 12 and 15, which were the source of the chromosome instability and MLL amplification, can be explained by telomere fusion events. The occurrence of two telomere fusion events in an otherwise typical MDS/AML karyotype could suggest that telomere erosion was significant in this patient's bone marrow cells, contributing to eventual amplification of $M L L$ via the BFB cycle, and disease progression.

FISH investigations have exposed the complexity of a disease process that evolved rapidly from RAEB-2 to AML, 


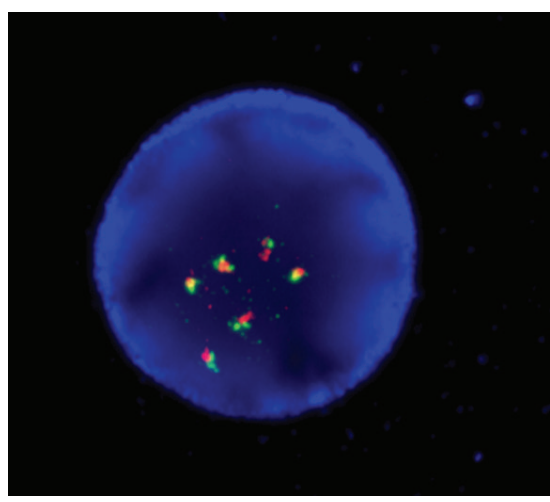

(a)

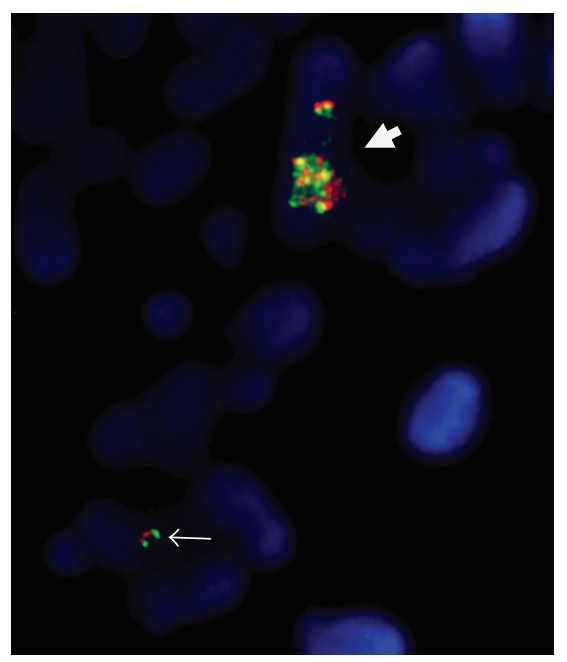

(b)

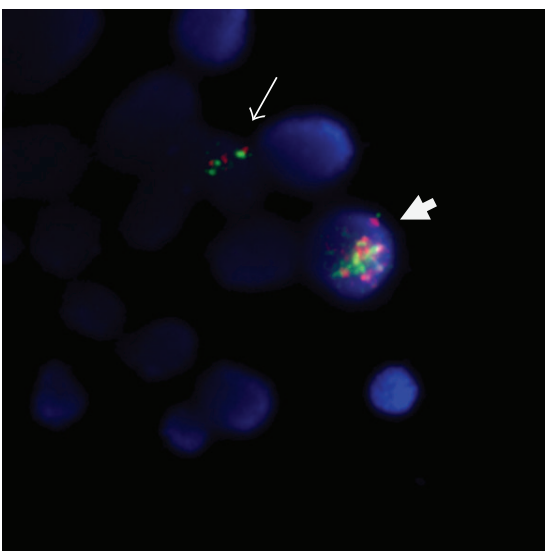

(c)

FIGURE 3: Representative images of FISH with the Vysis MLL Dual Color, Break Apart Rearrangement Probe. (a) interphase nucleus showing six clear $M L L$ fusion signals. (b) The der(11;15;12) (thick arrow) with multiple $M L L$ signals and a single fusion signal representing the normal chromosome 11. (c) The ring (11) (thick arrow) also with multiple MLL signals. In (b) and (c) the normal chromosome 11 is indicated by a thin arrow.

illustrating how loss of telomere function and BFB cycles appear to have played a major role in genomic instability leading to gene amplification and karyotypic complexity.

\section{Acknowledgment}

Ruth N. Mackinnon received support from the James and Vera Lawson Trust (ANZ Trustees).

\section{References}

[1] S. M. Bailey and J. P. Murnane, "Telomeres, chromosome instability and cancer," Nucleic Acids Research, vol. 34, no. 8, pp. 2408-2417, 2006.

[2] B. McClintock, "The stability of broken ends of chromosomes in Zea Mays," Genetics, vol. 26, no. 2, pp. 234-282, 1941.

[3] C. H. Jones, C. Pepper, and D. M. Baird, “Telomere dysfunction and its role in haematological cancer," British Journal of Haematology, vol. 156, no. 5, pp. 573-587, 2012.

[4] J. P. Murnane, "Telomere dysfunction and chromosome instability," Mutation Research, vol. 730, no. 1-2, pp. 28-36, 2012.

[5] A. W. I. Lo, L. Sabatier, B. Fouladi, G. Pottiert, M. Ricoul, and J. P. Murnane, "DNA amplification by breakage/fusion/bridge cycles initiated by spontaneous telomere loss in a human cancer cell line," Neoplasia, vol. 4, no. 6, pp. 531-538, 2002.

[6] B. T. Letsolo, J. Rowson, and D. M. Baird, "Fusion of short telomeres in human cells is characterized by extensive deletion and microhomology, and can result in complex rearrangements," Nucleic Acids Research, vol. 38, no. 6, pp. 1841-1852, 2009.

[7] D. Gisselsson, M. Höglund, F. Mertens et al., "The structure and dynamics of ring chromosomes in human neoplastic and nonneoplastic cells," Human Genetics, vol. 104, no. 4, pp. 315-325, 1999.
[8] S. Chakraborty, J. M. Stark, C. L. Sun et al., "Chronic myelogenous leukemia stem and progenitor cells demonstrate chromosomal instability related to repeated breakage-fusion-bridge cycles mediated by increased nonhomologous end joining," Blood, vol. 119, no. 26, pp. 6187-6197, 2012.

[9] J. P. Murnane, "Telomere loss as a mechanism for chromosome instability in human cancer," Cancer Research, vol. 70, no. 11, pp. 4255-4259, 2010.

[10] A. Mohamed, "MLL amplification in leukemia," 2011, http://atlasgeneticsoncology.org//Anomalies/MLLampliID 1547.html.

[11] K. C. Rayeroux and L. J. Campbell, "Gene amplification in myeloid leukemias elucidated by fluorescence in situ hybridization," Cancer Genetics and Cytogenetics, vol. 193, no. 1, pp. 44-53, 2009.

[12] M. K. Andersen and J. Pedersen-Bjergaard, "Increased frequency of dicentric chromosomes in therapy-related MDS and AML compared to de novo disease is significantly related to previous treatment with alkylating agents and suggests a specific susceptibility to chromosome breakage at the centromere," Leukemia, vol. 14, no. 1, pp. 105-111, 2000.

[13] R. N. MacKinnon, C. Patsouris, I. Chudoba, and L. J. Campbell, "A FISH comparison of variant derivatives of the recurrent $\operatorname{dic}(17 ; 20)$ of myelodysplastic syndromes and acute myeloid leukemia: obligatory retention of genes on $17 \mathrm{p}$ and $20 \mathrm{q}$ may explain the formation of dicentric chromosomes," Genes Chromosomes and Cancer, vol. 46, no. 1, pp. 27-36, 2007.

[14] B. A. Sullivan and H. F. Willard, "Stable dicentric X chromosomes with two functional centromeres," Nature Genetics, vol. 20, no. 3, pp. 227-228, 1998.

[15] R. N. MacKinnon and L. J. Campbell, "A comparison of two contrasting recurrent isochromosomes 20 found in myelodysplastic syndromes suggests that retention of proximal 20q is a significant factor in myeloid malignancies," Cancer Genetics and Cytogenetics, vol. 163, no. 2, pp. 176-179, 2005.

[16] W. C. Earnshaw and B. R. Migeon, "Three related centromere proteins are absent from the inactive centromere of a stable 
isodicentric chromosome," Chromosoma, vol. 92, no. 4, pp. 290296, 1985.

[17] L. G. Schaffer, M. L. Slovak, and L. J. Campbell, Eds., ISCN 2009: An International System for Human Cytogenetic Nomenclature, Karger, Basel, Switzerland, 2009.

[18] R. W. Frenck Jr., E. H. Blackburn, and K. M. Shannon, “The rate of telomere sequence loss in human leukocytes varies with age," Proceedings of the National Academy of Sciences of the United States of America, vol. 95, no. 10, pp. 5607-5610, 1998.

[19] D. Gisselsson, L. Pettersson, M. Höglund et al., "Chromosomal breakage-fusion-bridge events cause genetic intratumor heterogeneity," Proceedings of the National Academy of Sciences of the United States of America, vol. 97, no. 10, pp. 5357-5362, 2000.

[20] B. Britt-Compton, T. T. Lin, G. Ahmed et al., "Extreme telomere erosion in ATM-mutated and 11q-deleted CLL patients is independent of disease stage," Leukemia, vol. 26, no. 4, pp. 826830, 2012.

[21] R. T. Calado, J. N. Cooper, H. M. Padilla-Nash et al., "Short telomeres result in chromosomal instability in hematopoietic cells and precede malignant evolution in human aplastic anemia," Leukemia, vol. 26, no. 4, pp. 700-707, 2012.

[22] P. J. Campbell, S. Yachida, L. J. Mudie et al., "The patterns and dynamics of genomic instability in metastatic pancreatic cancer," Nature, vol. 467, no. 7319, pp. 1109-1113, 2010.

[23] G. Leone, L. Fianchi, L. Pagano, and M. T. Voso, "Incidence and susceptibility to therapy-related myeloid neoplasms," ChemicoBiological Interactions, vol. 184, no. 1-2, pp. 39-45, 2010. 


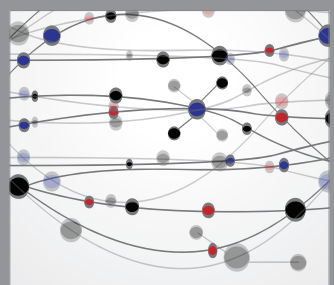

The Scientific World Journal
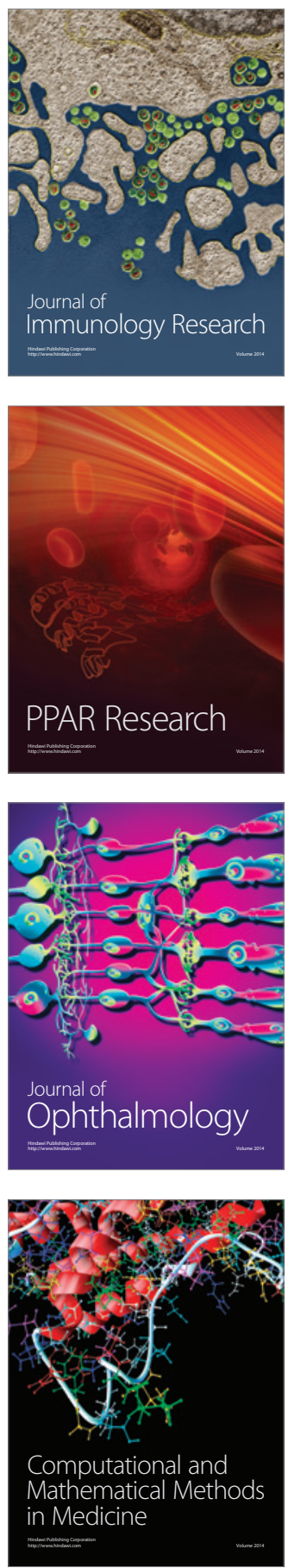

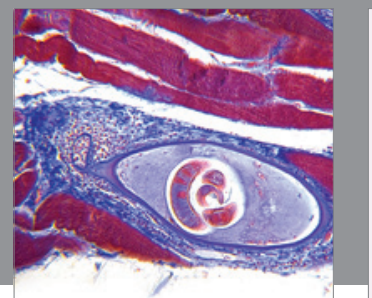

Gastroenterology

Research and Practice
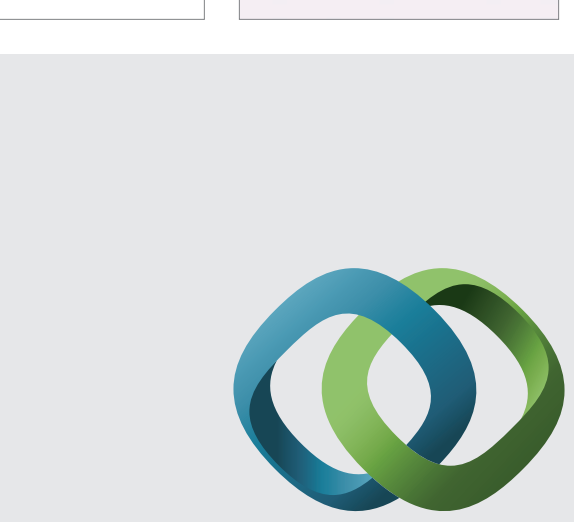

\section{Hindawi}

Submit your manuscripts at

http://www.hindawi.com
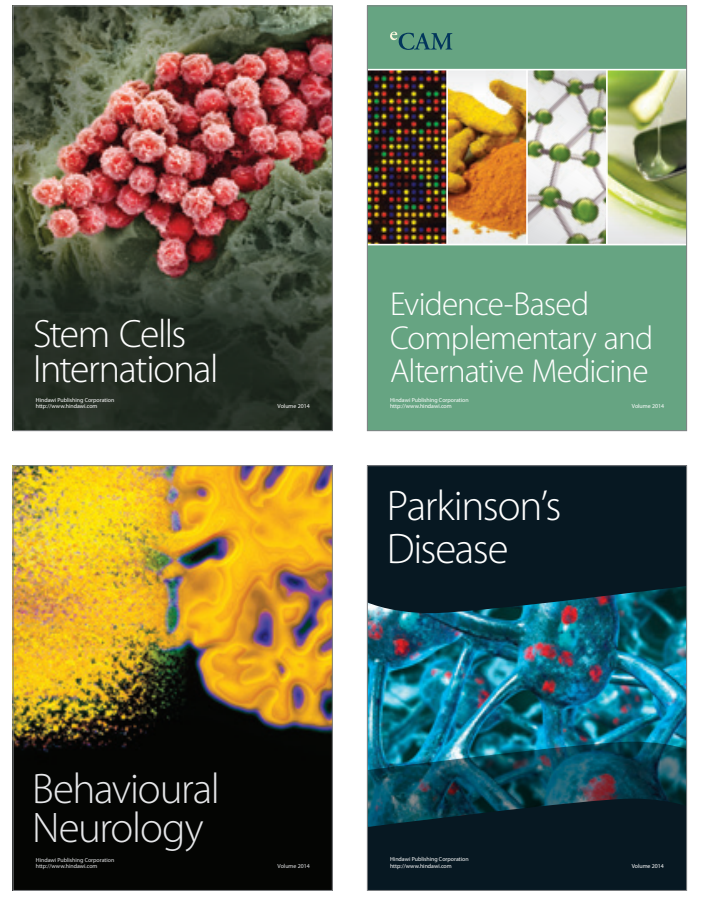
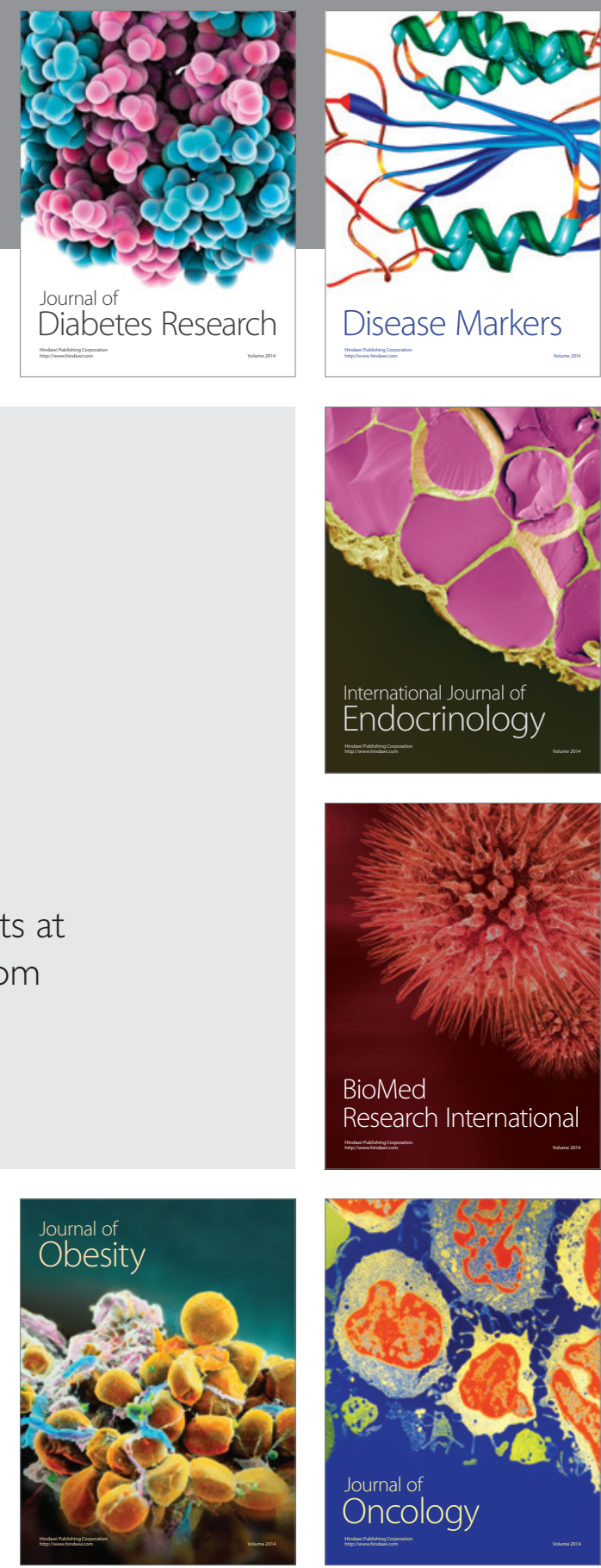

Disease Markers
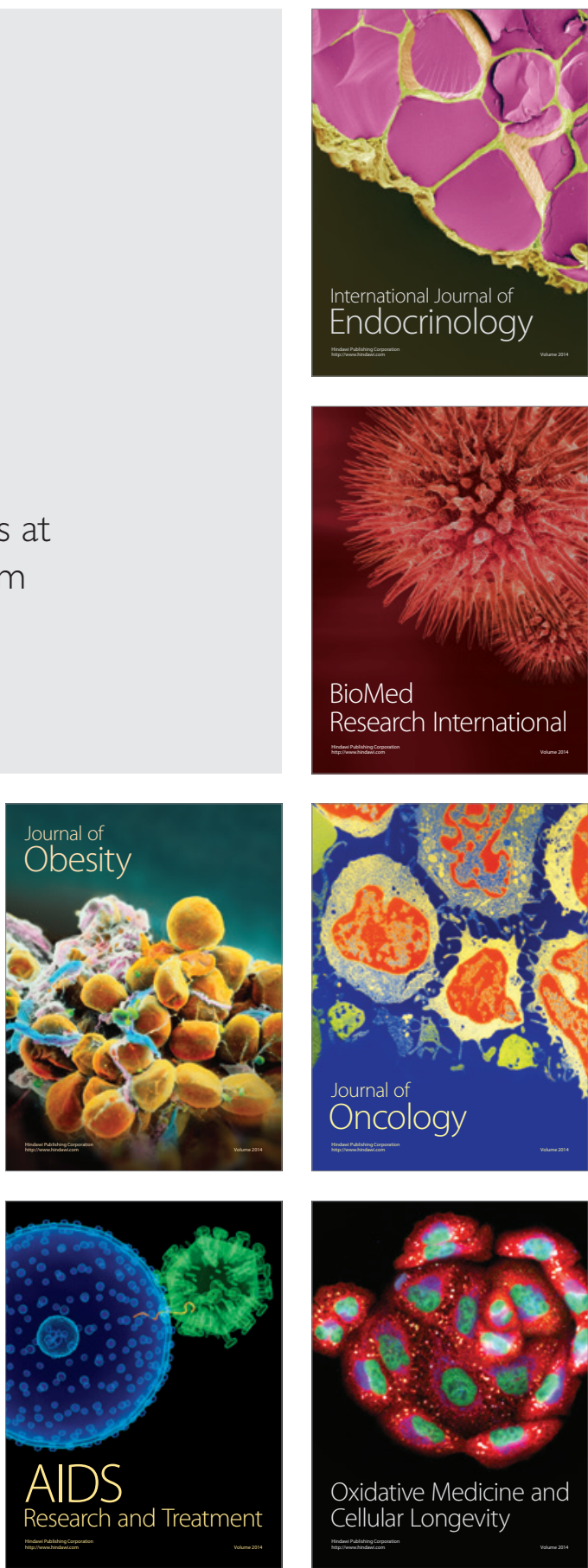